\title{
Ecología de medios, imaginarios y algoritmos: Una mirada sobre las dinámicas comunicativas del activismo digital
}

\author{
Autor: Emiliano Treré \\ Título: Hybrid media activism. Ecologies, imaginaries, algorithms \\ Año: 2019 \\ Ciudad: Londres / Nueva York \\ Editorial: Routledge \\ ISBN: 978-1-138-21814-7 \\ 236 págs.
}

\section{Antecedentes}

Hybrid media activism. Ecologies, imaginaries, algorithms es una de las incursiones más estimulantes y completas al universo complejo y no menos contradictorio del activismo digital. Un libro que ofrece, por una parte, un dilatado esfuerzo por revisar, sintetizar y discutir las principales aproximaciones teóricas al campo de la comunicación digital y de la acción colectiva. Simultáneamente, nos presenta un completo trabajo empírico que aborda y sistematiza las dinámicas comunicativas y organizativas de algunos de los principales movimientos sociales de la última década (Onda Anómala y el Movimiento 5 Estrellas —en Italia-, \#YoSoyl32 — México- y el 15M España-). El diálogo y la yuxtaposición que hace Treré entre estos dos planos - el teórico y el empírico - le permiten desarrollar un enfoque que se aleja de las perspectivas más dogmáticas para poner en valor la complejidad y la ambivalencia de la tecnología y de las prácticas comunicativas contemporáneas. Esto hace que Hybrid media activism sea capaz de recoger, de manera matizada y contrastada, tanto las oportunidades expresivas que encuentran los colectivos sociales como los riesgos y limitaciones que enfrentan en el escenario comunicativo actual.

\section{Ecologías, imaginarios y algoritmos}

Treré comienza cuestionando el reduccionismo de una serie de planteamientos teóricos sobre comunicación y movimientos sociales. Concretamente critica 
"tres espectros": 1. La visión instrumental de la tecnología, 2. La perspectiva funcionalista y su escasa atención a la identidad colectiva, y 3. El determinismo tecnológico; y “cinco falacias": 1. La separación dualista entre lo online y lo offline, 2. El análisis aislado de medios o plataformas digitales, 3. El presentismo, 4. La "miopía de lo visible" (Melucci, 1988), que sobredimensiona lo cuantificable e invisibiliza los procesos comunicativos y organizativos, y 5. La asunción acrítica de la tecnología digital.

Para superar dicho reduccionismo, Treré contribuye a revitalizar la "Teoría de la práctica" (Giddens, 1984; Bourdieu, 1990; Schatzki, 1996) y a profundizar en su aplicación al ámbito de la comunicación — siguiendo a Couldry (2004)—y de los movimientos sociales. Específicamente, considera que al hablar de prácticas comunicativas hemos de atender a tres dimensiones interrelacionadas: 1 . La naturaleza compleja, híbrida y multifacética de la actual ecología de medios, 2. Los procesos de creación de significados, que incluyen la formación de identidades colectivas, imaginarios y mitologías, y 3. La importancia de la materialidad y de su impacto en las dinámicas sociales.

Para abordar estos tres aspectos, recurre a tres conceptos clave: "ecologías", "imaginarios" y "algoritmos", que funcionan como punto de partida del libro y que vertebran su estructura y su exposición de resultados empíricos.

En el primer apartado del libro — “Ecologías” - se revisa y evalúa las principales aportaciones de los enfoques analíticos basados en la "ecología de medios" (Mattoni, 2017). Estos enfoques sirven para superar el reduccionismo que representan las "cinco falacias" citadas previamente, contraponiéndoles un enfoque "holístico" (Treré, 2012, 2019) que atienda a:

1. La multiplicidad de plataformas y tecnologías con las que interaccionan los movimientos sociales.

2. Los procesos de hibridación entre lo offline y lo online, entre lo nuevo y lo viejo, lo corporativo y lo alternativo, lo interno y lo externo, lo humano y lo no humano.

3. La complejidad de las prácticas de los movimientos, más allá de los productos comunicativos finales. Así como a su relación con los contextos socioculturales concretos.

4. La acumulación de competencias y aprendizajes con relación a las tecnologías y a sus posibles apropiaciones por parte de la sociedad civil.

5. La importancia de mantener una aproximación crítica hacia el uso de determinadas tecnologías, atendiendo a sus lógicas corporativas específicas.

En el segundo apartado - "Imaginarios" - se ofrece un extenso recorrido teórico, que recoge la conceptualización de los "imaginarios sociales" (Castoriadis, 1998; Anderson, 1991; Taylor, 2004) como un mundo de significaciones e ideas, pero también como el marco de referencia en el que 
toman forma las "prácticas" sociales (Herman, 2010) y en el que tienen lugar procesos colectivos de "imaginación radical" (Haiven \& Khasnabish, 2014).

En esta segunda sección, por tanto, se cuestiona los mitos y relatos hiperbólicos que han acompañado al desarrollo de las tecnologías digitales en las últimas décadas. Relatos deterministas que han ido renovándose cíclicamente (Mosco, 2004) ante cada transformación de calado (de la web 2.0 a los procesos de datafiacción), y que están vinculados, principalmente, al fetichismo tecnológico y al tecnoutopismo, pero también a los imaginarios distópicos respecto a las tecnologías.

Finalmente, en la sección "Algoritmos" se adentra en la relación dialéctica entre tecnologías y agentes sociales. Se muestra cómo las dinámicas comunicativas y organizativas de los movimientos son reconfiguradas por los modelos de propiedad y el diseño de las tecnologías, así como por los procesos de dataficación. Pero, al mismo tiempo, se visibiliza cómo la agencia individual y colectiva genera dinámicas de apropiación y reprogramación de las infraestructuras y plataformas digitales, permitiendo el despliegue de prácticas de resistencia algorítmica y de activismo de datos (Milan, 2017).

Concretamente, se analizan las estrategias de sabotaje, manipulación y represión puestas en práctica por el PRI y por seguidores de Enrique Peña Nieto en México a través de plataformas digitales, y las prácticas de resistencia que tanto en el contexto mexicano como en el caso español fueron capaces de desarrollar las organizaciones sociales. En este sentido, Treré reconoce la capacidad de movimientos como el $15 \mathrm{M}$ para entender las oportunidades políticas que ofrecían los algoritmos y su capacidad para adaptar sus estrategias comunicativas al diseño de las plataformas utilizadas. Pero también reconoce que el éxito de estas estrategias depende, en buena medida, de contar con un contexto social y político favorable, donde otros actores institucionales no estén implementando modelos similares para controlar el escenario comunicativo digital.

Podríamos decir que el surgimiento de esos agentes institucionales grupos vinculados a partidos políticos, a agencias de comunicación o a sectores gubernamentales - forma parte de las tendencias que han ido reproduciéndose en diferentes latitudes durante los últimos años. Al respecto, destacan las estrategias de desinformación de la Alt-right y de su franquicia internacional o las tácticas de desestabilización y polarización implementadas por compañías como Internet Research Agency a escala internacional (Peirano, 2019). En este sentido, Treré anticipa buena parte de estas prácticas y contribuye a superar las visiones instrumentales de las tecnologías, poniendo de relieve la importancia de las dinámicas adoptadas por los actores sociales e institucionales. 


\section{Discusión}

En definitiva, Hybrid media activism. Ecologies, imaginaries, algorithms es una contribución destacada al dilatado esfuerzo por cartografiar el activismo digital. Un trabajo que se aleja de la urgencia y el efectismo para condensar más de 10 años de aproximaciones empíricas y de refinamiento conceptual. Una pieza clave para ampliar nuestra comprensión, sin dogmatismos ni maximalismos, de la complejidad de las prácticas sociales y comunicativas contemporáneas, tan multifacéticas, ambivalentes, $\mathrm{y}$, por qué no, indisciplinadas.

Francisco Javier López-Ferrández

Doctor en Estudios Internacionales de Paz, Conflictos y Desarrollo por la Universitat Jaume I (España)

\section{Referencias bibliográficas}

Anderson, B. (1991). Imagined communities: reflections on the origin and spread of nationalism. Londres: Verso.

Bourdieu, P. (1990). The logic of practice. Stanford: Stanford University Press.

Castoriadis, C. (1998). The Imaginary Institution of Society. Cambridge: MIT Press.

Couldry, N. (2004). Theorising media as practice. Social Semiotics, 14 (2), 115-132.

Giddens, A. (1984). The constitution of society: outline of the theory of structuration. Berkeley / Los Angeles: University of California Press.

Haiven, M., \& Khasnabish, A. (2014). The radical imagination: social movement research in the age of austerity. Londres: Zed Books.

Herman, A. (2010). The network we all dream of: manifest dreams of connectivity and communication or, Social Imaginaries of the wireless commons. En B. Crow, B. Longford, $\&$ K. Sawchuk (Eds.), The wireless spectrum: the politics, practices, and poetics of mobile media. Toronto: University of Toronto Press.

Mattoni, A. (2017). A situated understanding of digital technologies in social movements. Media ecology and media practice approaches. Social Movement Studies, 16(4), 494-505.

Melucci, A. (1988). Getting involved: identity and mobilization in social movements. International Social Movement Research, 1, 329-348.

Milan, S. (2017). Data activism as the new frontier of media activism. En V. W. Pickard, \& G. Yang (Eds.), Media activism in the digital age. Abingdon: Routledge.

Mosco, V. (2004). The digital sublime: myth, power, and cyberspace. Cambridge / Londres: MIT Press.

Peirano, M. (2019). El enemigo conoce el sistema. Madrid: Debate.

Schatzki, T. (1996). Practiced bodies: subjects, genders and minds. En T. Schatzki, \& W. Natter (Eds.), The social and political body. Nueva York: Guilford Press.

Taylor, C. (2004). Modern social imaginaries. Londres: Duke University Press.

Treré, E. (2012). Social movements as information ecologies: Exploring the coevolution of multiple internet technologies for activism. International Journal of Communication, 6, 2359-2377.

Treré, E. (2019). Hybrid media activism. Ecologies, imaginaries, algorithms. Londres / Nueva York: Routledge. 